\title{
An MR-Compatible Optical Force Sensor for Human Function Modeling
}

\author{
Mitsunori Tada ${ }^{1,2}$ and Takeo Kanade ${ }^{1,2,3}$ \\ 1 Digital Human Research Center \\ National Institute of Advanced Industrial Science and Technology \\ 2-41-6, Aomi, Koto-ku, Tokyo 135-0064, Japan. \\ m.tada@aist.go.jp \\ 2 CREST, Japan Science and Technology Agency \\ 3 The Robotics Institute, Carnegie Mellon University \\ 5000 Forbes Avenue, Pittsburgh, PA 15213, USA. \\ tk@cs.cmu.edu
}

\begin{abstract}
This paper presents the principle, structure and performance of a newly developed MR-compatible force sensor. It employs a new optical micrometry that enables highly accurate and highly sensitive displacement measurement. The sensor accuracy is better than $1.0 \%$, and the maximum displacement of the detector is about $10 \mu \mathrm{m}$ for a range of the applied force from 0 to $6 \mathrm{~N}$.
\end{abstract}

\section{Introduction}

Magnetic resonance imaging (MRI) has widely been used in studies of human function because it is non-invasive and has high spatial resolution. However, since MRI requires homogeneous magnetic field and radio frequency (RF) pulse for inducing the nuclear magnetic resonance (NMR), standard mechanical and electrical devices, composed of ferromagnetic materials and electrical circuits, cannot be used in the imaging volume of MRI [1].

The development of mechatronical sensor devices that do not interfere with MRI is one of the key technologies 23] in experiments using MRI, especially for studies such as human anatomy [4 and brain functional mapping during motor control and somatosensory perception [5, so that we can quantify the applied load and the voluntary human movement simultaneously while imaging.

Some MR-compatible force sensors have been proposed. Liu et al. have developed a hydraulic grip dynamometer 6]. The grip force applied to a hand grip device is transmitted to a pressure transducer outside MRI by a water-filled nylon tube. Due to the friction of a piston built into the hand grip device, the grip dynamometer has a dead zone over a wide range of force, from 0 to $80 \mathrm{~N}$.

We have recently proposed an MR-compatible force sensor 78 based on an optical micrometry 9 using an LED and a quadrant photo diode. It achieves MR compatibility by placing both optical elements outside the MRI and extending a light path between them by using fiber optics. It was found, however, that in this design, since the light emitting fiber is fixed on the force detector of the 


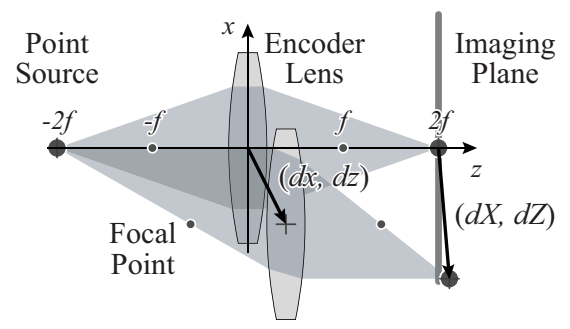

Fig. 1. Principle of the proposed optical micrometry

sensor, mechanical oscillation of the optical fiber easily causes perturbation of the force measurement.

This paper presents a newly designed and developed MR-compatible force sensor that has eliminated the above problem: its principle, structure, and performance. A new optical micrometry is employed that enables highly accurate and highly sensitive displacement measurement. The measurement accuracy is better than $1.0 \%$ and the maximum displacement of the detector is about $10 \mu \mathrm{m}$ under the applied force ranging from 0 to $6 \mathrm{~N}$. The accuracy and rigidity are comparable to commercially available non-MR-compatible force sensors.

\section{Two-Dimensional Optical Micrometry}

\subsection{Principle}

Figure 1 illustrates the principle of the new optical micrometry for measuring minute displacement along one-dimensional direction $x$ perpendicular to the direction of the optical path (along $z$ ). The coordinate origin is placed at the position where an optical lens with focal length $f$, called an encoder lens, is at the rest. A point light source is positioned at $(0,-2 f)$, and an imaging plane at $(0,2 f)$. The point source is focused onto the center of the imaging plane $(0,2 f)$ when the lens is at the origin.

When a minute translational displacement $(d x, d z)$ is applied to the encoder lens, it results in a shift of the focused image of the point source by $(d X, d Z)$,

$$
(d X, d Z)=\left(2 d x-\frac{d x d z}{f+d z}, \frac{d z^{2}}{f+d z}\right)
$$

Neglecting higher order terms,

$$
(d X, d Z)=(2 d x, 0)
$$

Note that the lens has magnified $x$ axial displacement $d x$ two times onto the change in the imaging plane, whereas nullifying the effect of $z$ axial displacement $d z$. The shift of the focused image location can be measured accurately by using a position detectable photo sensor, such as a segmented photo diode or a position 

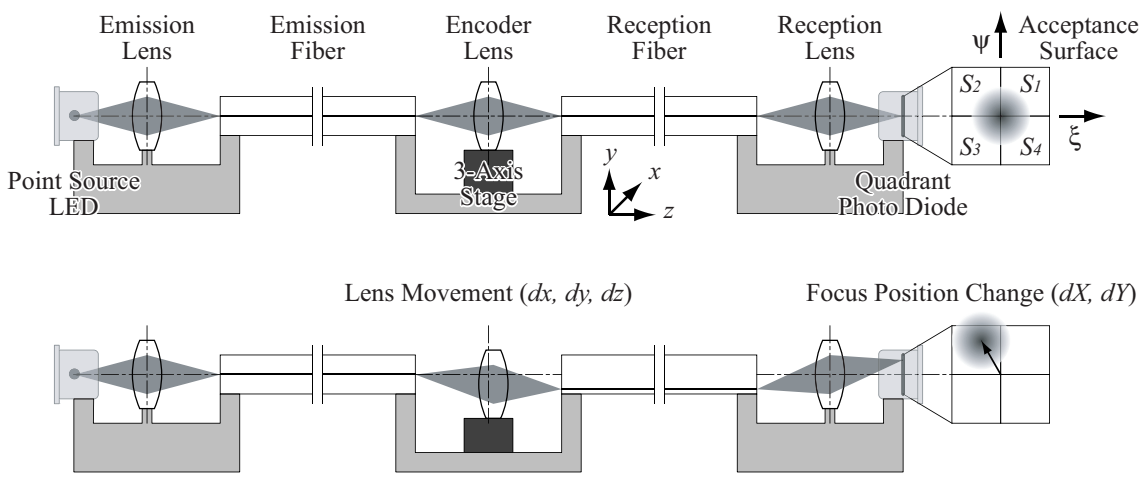

Fig. 2. Experimental set-up for the evaluation of the displacement measurement

sensitive device (PSD). The one-dimensional optical micrometry described above can be generalized to a two-dimensional case; lens displacement within the $x-y$ plane perpendicular to the optical axis $(\mathrm{z})$ can be measured using a quadrant photo diode or a two-dimensional PSD.

It should also be noted that the point source and the imaging plane do not move and can be mechanically connected to the same base, and the encoder lens displaces with respect to the base. In the MRI environment where ferromagnetic materials or electronic circuits are severely restricted, all the electric devices on the base component should be positioned apart from the encoder lens. Such a configuration can be realized by using multi-cored fiber optics to connect a light path from the actual point source to the point source in Fig. 1, and to connect the imaging plane in Fig. 1 to the actual photo detector.

This configuration has distinct advantages over the conventional design [78] in which the light source and the sensing device are placed on separate bases and their relative displacement is measured. Firstly, displacement is magnified by a factor of two (higher precision). Secondly, the encoder lens movement along the optical axis has little influence on the displacement measurement (axial independence). Thirdly and equally important for our purpose, there is no need for fiber to be connected to the vibrating component. As a result, oscillation of the fiber optics has no or little effect on the measurement (mechanical independence).

\subsection{Experimental Set-Up}

We have evaluated the accuracy of the two-dimensional micrometry. Shown in Fig. 2 is the schematic diagram of an experimental set-up constructed for this purpose. Light signal emitted from a point-source red LED (Alpha-One Electronics Co., VS679TM) is condensed by the emission lens (Nihon Sheet Glass Co., W18-S0290-063-ABC) and enters the multi-cored emission fiber (Keyence Co., FU-77) whose end-faces are carefully polished. The light emitted from the other end of the emission fiber, is condensed again by the encoder lens (the same 


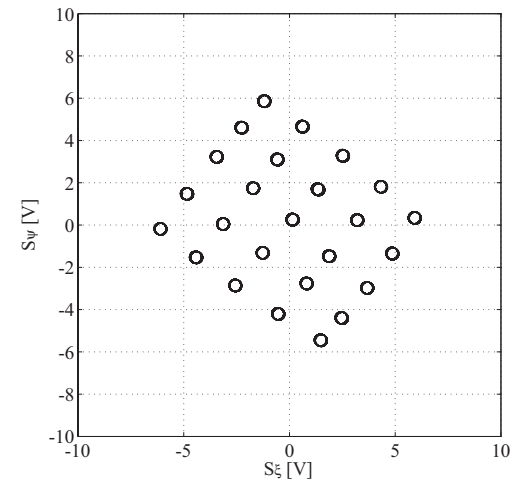

(a) Outputs of the amplifier

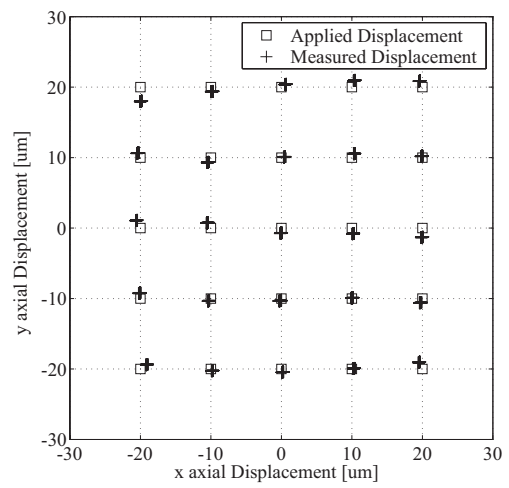

(b) Applied and measured displacements

Fig. 3. Evaluation of the two-dimensional optical micrometry

model as the emission lens). The encoder lens is mounted on a three-axis block stage (Sigma Koki Co., TSD-405C), and thus its minute displacement causes the lens to move minutely. As derived in equation (2), translational displacement of the encoder lens results in a shift of the focus location.

The light distribution of imaging plane is transmitted through the reception fiber (the same model as the emission fiber) with multiple cores. Light signal emitted from the other end of the reception fiber is condensed by the reception lens (the same model as the emission lens), and is finally focused onto the quadrant photo diode surface (Moririka Co., MI-1515H-4D). Both emission and reception optical fibers are 10 meters long and have 217 cores.

The output current from each photo diode segment is amplified with the I-V converter composed of an operational amplifier (Analog Devices Inc., OP400). The outputs from these four photo diode segments are fed to instrumentation amplifiers (Texas Instruments Inc., INA105), which compute,

$$
\left\{\begin{array}{l}
S_{\xi}=\left(S_{1}+S_{4}\right)-\left(S_{2}+S_{3}\right) \\
S_{\psi}=\left(S_{1}+S_{2}\right)-\left(S_{3}+S_{4}\right)
\end{array}\right.
$$

If the shift of the image of the point source is sufficiently minute, $(d X, d Y)$ and $\left(S_{\xi}, S_{\psi}\right)$ are related with a linear transformation, and then $(d x, d y)$ is obtained by the two-dimensional version of equation (2).

\subsection{Evaluation}

Accuracy of the two-dimensional micrometry was examined by the following procedure.

1. Move the encoder lens within a plane perpendicular to the optical axis, find the position at which both amplifier outputs, $S_{\xi}$ and $S_{\psi}$, become nearly zero, and define that position as the sensor origin. 


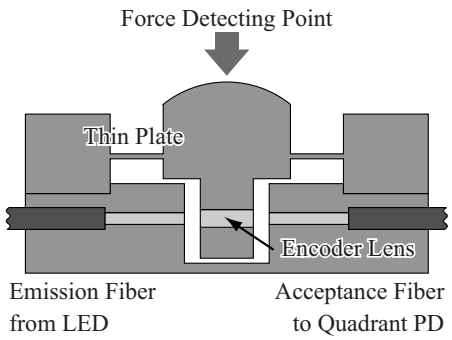

(a) Cross sectional view

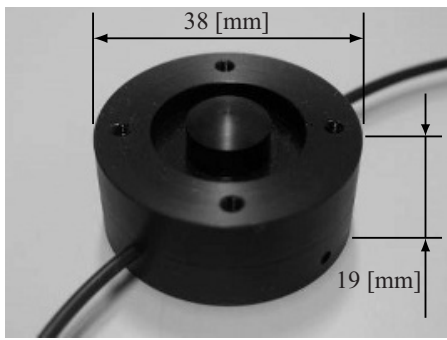

(b) External view

Fig. 4. Structure of the developed optical force sensor

2. Move the encoder lens at $5 \times 5$ grid positions $(0, \pm 10$ and $\pm 20 \mu \mathrm{m})$, centered at the sensor origin. At each of 25 positions, outputs of the amplifier are sampled 100 times.

Figure 3 -(a) plots the outputs of the differential amplifier at the grid positions. It shows the 100 data samples. Because of the relative rotation between the axes of the three-axis stage and the axes of the quadrant photo diode, there is rotation between the $x-y$ space and the $\xi-\psi$ space.

The lens displacement is modeled by a linear transformation of the outputs of the differential amplifier.

$$
\left(\begin{array}{c}
d x \\
d y \\
1
\end{array}\right)=\left(\begin{array}{ccc}
c_{11} & c_{12} & c_{13} \\
c_{21} & c_{22} & c_{23} \\
0 & 0 & 1
\end{array}\right)\left(\begin{array}{c}
S_{\xi} \\
S_{\psi} \\
1
\end{array}\right)=\boldsymbol{C}_{m}\left(\begin{array}{c}
S_{\xi} \\
S_{\psi} \\
1
\end{array}\right)
$$

The calibration matrix $\boldsymbol{C}_{m}$ is estimated by least square method using reference data set. Figure 3.(b) shows the result; the relation between the applied displacement and the measured displacement. It also shows the 100 data samples. While there is distortion caused by the large lens displacement in the upper left of the square, the accuracy of the measurement is better than $1.0 \%$ at the other points. We can confirm high linearity, high axial independency and high repeatability of the proposed micrometry from this plot.

\section{MR-Compatible Force Sensor}

We have designed an MR-compatible force sensor that uses the optical micrometry described in the previous section for displacement sensing. We have evaluated its accuracy and sensitivity in force measurement, and confirmed its MR compatibility.

\section{$3.1 \quad$ Structure}

Figure 4 (a) shows the cross sectional view of the force sensor. The force detector at the center is supported by a $0.4 \mathrm{~mm}$-thick annular plate. It converts the 


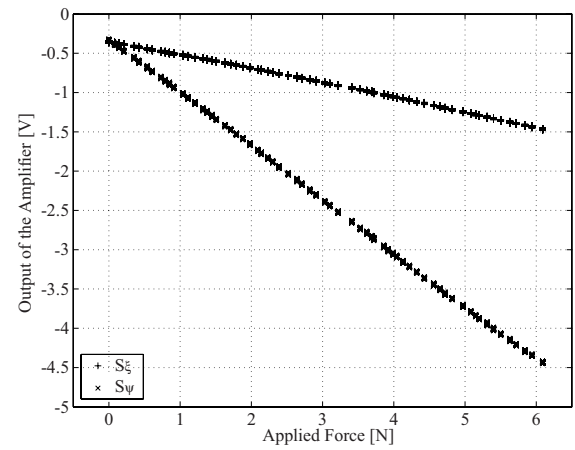

(a) Outputs of the amplifier

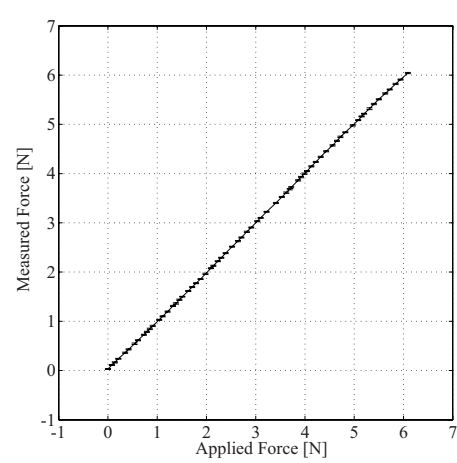

(b) Applied and measured force

Fig. 5. Evaluation of the developed optical force sensor

applied force (downward direction in this figure) into minute displacement of the encoder lens that is connected to it at the bottom. For the encoder lens, we use a gradient index (GRIN) lens (Nihon Sheet Glass Co., W18-S0290-063-ABC) because its small dimension and short focal length help reduce the entire size of the force sensor.

Shown in Fig. 4t-(b) is the external view of the force sensor. The sensing module is $38 \mathrm{~mm}$ in diameter and $19 \mathrm{~mm}$ in height. It is made of a polyoxymethylene resin to reduce the affect of the magnetic field and the RF pulse of MRI. Note that this sensor head component corresponds to the middle part in Fig. 2, which is free from any magnetic or electronic elements.

Other optical components (including the fiber optics, LED, photo diode and optical lenses) and the differential amplifier are the same as those of the experimental set-up described in 2.2 except that the light source and the quadrant photo diode are supported by small, two-axis block stages (Sigma Koki Co., TASB-152) for zero-alignment of the optical axis.

\subsection{Evaluation}

Figure 54(a) plots the outputs of the differential amplifier with respect to the applied force. It shows the 100 data samples collected in each condition. Figure 5. (b) shows the relation between the applied force and the measured force. It shows the mean and variance of the 100 data samples. The accuracy is better than $1.0 \%$. The maximum displacement of the encoder lens is less than $10 \mu \mathrm{m}$ under the applied force ranging from 0 to $6 \mathrm{~N}$.

We have confirmed that the accuracy and the rigidity of the developed force sensor is equivalent to that of commercially available force sensors. However, since the sensor module is made of a polyoxymethylene resin, hysteresis cannot be ignored if the applied force is greater than the rated force. Furthermore, the natural frequency of the force sensor is about ten times lower compared with the force sensors made of metallic materials. 


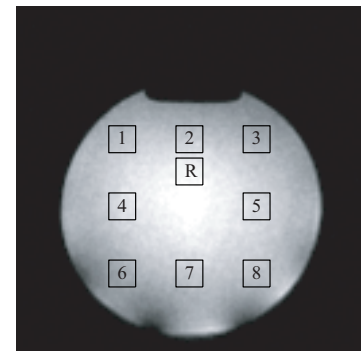

(a) Normal phantom

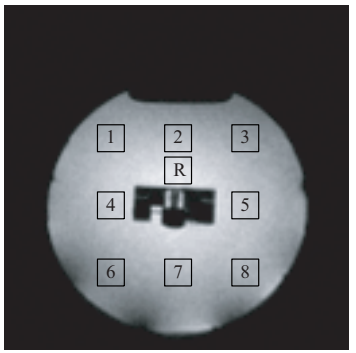

(b) Sensor-installed phantom

Fig. 6. Cross sectional images of cylindrical phantoms

Table 1. Signal to noise ratio (SNR) of the obtained images and SNR losses induced by the force sensor

\begin{tabular}{l|r|r|r|r|r|r|r|r} 
Window number & 1 & 2 & 3 & 4 & 5 & 6 & 7 & 8 \\
\hline \hline SNR (Normal phantom) & 20.65 & 15.27 & 18.26 & 23.81 & 35.18 & 20.99 & 20.22 & 26.52 \\
\hline SNR (Sensor-installed phantom) & 20.40 & 14.80 & 17.93 & 23.57 & 34.28 & 20.42 & 19.69 & 26.13 \\
\hline \hline SNR loss (\%) & 1.21 & 3.11 & 1.77 & 1.03 & 2.55 & 2.72 & 2.63 & 1.45
\end{tabular}

\subsection{Evaluation of MR Compatibility}

We have evaluated the loss of homogeneity of the magnetic field and the losses of signal-to-noise ratio (SNR) of the obtained images caused by the insertion of the force sensor. Two types of phantoms were imaged using $2 \mathrm{~T}$ experimental MRI with $310 \mathrm{~mm}$ bore: one is a normal phantom that is a cylindrical plastic bottle filled with deionized water; and the other is a sensor-installed phantom that is the same as the normal phantom except that the force sensor is installed at the center. The phantoms were placed at the center of the MRI bore. The imaging sequence is gradient echo, TE/TR $=5 / 500 \mathrm{msec}, \mathrm{FOV}=160 \times 160 \mathrm{~mm}$, and slice thickness is $2 \mathrm{~mm}$.

The inhomogeneity is defined by the diversity of the observed spectrum. Inhomogeneity of the magnetic field caused by the insertion of the force sensor is $0.61 \mathrm{ppm}$, which is almost identical to the inhomogeneity $0.54 \mathrm{ppm}$ when the normal phantom is placed.

Figure 6-(a) and (b) show MR images of the normal phantom and the sensorinstalled phantom, respectively. In both figures, rectangular image windows for calculation of the SNR are also shown. There is no apparent distortion in the image of the sensor-installed phantom. The SNR is calculated using the following equation,

$$
S N R=m_{r} / \sigma
$$


where $m_{r}$ is the mean value of the $10 \times 10$ pixel reference window, and $\sigma$ is the standard deviation of the $10 \times 10$ pixel test window. Though, ideally the reference window should be placed at the center of the phantom, it was selected a little above the center as shown $\mathrm{R}$ in Fig. 6 in order to avoid including pixels of the sensor. Test windows are 1 to 8 . Table 1 shows calculated SNR values and their losses caused by the force sensor. The observed SNR losses are 1.03 to $3.11 \%$, which are sufficiently lower than the maximum acceptable SNR loss, $10 \%$ [3].

Furthermore, the collected force data during these evaluations are undisturbed by the magnetic field and the RF pulses. These results indicate that MRI has little influence on the sensor, and also the force sensor has little influence on the imaging sequence of MRI. The developed force sensor is confirmed to be highly MR-compatible.

\section{Conclusion}

An MR-compatible force sensor based on the new optical micrometry has been developed. Results of the evaluation have indicated that the micrometry is accurate and sensitive enough to be used for force sensing. Correspondingly, the developed force sensor is accurate, rigid and MR-compatible. We expect that the sensor is useful for studying human's force in the area of anatomical and brain functions using MRI.

The authors would gracefully acknowledge Dr. Homma for his assists in conducting experiments for evaluation of MR compatibility.

\section{References}

1. Chinzei, K., et al.: MR compatibility of mechatronic devices: Design criteria. In: Proc. of Second Int'l Conf. on MICCAI. (1999) 1020-1031

2. Masamune, K., et al.: Development of an MRI-compatible needle insertion manipulator for stereotactic nuerosurgery. J. of Image Guided Surgery 1 (1995) 242-248

3. Chinzei, K., et al.: MR compatible surgical assist robot: system integration and preliminary feasibility study. In: Proc. of Third Int'l Conf. on MICCAI. (2000) 921-930

4. Chenevert, T.L., et al.: Elasticity reconstructive imaging by means of stimulated echo MRI. J. of Magn. Reson. Med. 39 (1998) 482-490

5. Ehrsson, H.H., et al.: Cortical activity in precision-versus power-grip tasks: An fMRI study. J. of Neurophysiol. 83 (2000) 528-536

6. Liu, J.Z., et al.: Simultaneous measurement of human joint force, surface electromyograms, and functional MRI-measured brain activation. J. of Neurosciece Method 101 (2000) 49-57

7. Tada, M., et al.: Development of an optical 2-axis force sensor usable in MRI environments. In: Proc. of Int'l Conf. on Sensors. (2002)

8. Takahashi, N., et al.: An optical 6-axis force sensor for brain function analysis using fMRI. In: Proc. of Int'l Conf. on Sensors. (2003)

9. Hirose, S., et al.: Development of optical 6-axial force sensor and its signal calibration considering non-linear interference. In: Proc. of Int'l Conf. on Advanced Robotics. (1990) 46-53 